\title{
Response Inhibition Modulates Response Conflict in Task Switching
}

\author{
Kai Robin Grzyb and Ronald Hübner \\ Department of Psychology, University of Konstanz, Germany
}

\begin{abstract}
Although response repetition (RR) effects vary considerably between conditions and studies, little is known about the causes. Recently, RR costs on task switch trials have been found to be larger for incongruent stimuli that activate both alternative responses than for neutral ones. Here, we investigated if this modulation can be explained by an amplification of response conflict account (ARC). It assumes that a response shift bias that is responsible for the basic RR costs amplifies the response conflict induced by incongruent stimuli specifically on trials where the response repeats. Consequently, RR costs are increased for incongruent stimuli. Because supporting evidence for this account was restricted to task shift trials, we tested if the ARC account holds also more generally, that is, on task repetition trials. To this end, we applied a rather common alternating runs paradigm and presented neutral and incongruent stimuli. Results show that the congruency effect was larger on RR trials than on RS trials. Because this relation was independent of task transition, it is consistent with the idea that, in order to promote behavioral flexibility in task switching contexts, a general response shift bias is induced by inhibiting the previous response.
\end{abstract}

Keywords: response repetition, response inhibition, task switching, response conflict

When participants alternate between several tasks with overlapping responses, a characteristic relation can be observed between the effects of task transitions and those of response transitions. On task-repetition trials the repetition of a response usually leads to a performance advantage compared to a response shift (RS), whereas on task-switch trials response repetition (RR) produces costs (Druey \& Hübner, 2008; Hübner \& Druey, 2006; Kleinsorge \& Heuer, 1999; Koch, Schuch, Vu, \& Proctor, 2011; Meiran, 2000a; Rogers \& Monsell, 1995; Schuch \& Koch, 2004). If one considers the corresponding literature, it is obvious, though, that the interaction between response transition and task transition largely varies in size across studies and conditions (cf. Altmann, 2011).

The origin of this variation has recently been investigated by Grzyb and Hübner (2012a). In their study they specifically examined the role of stimulus congruency by presenting either neutral or incongruent stimuli. Whereas neutral stimuli were associated only with the correct response, incongruent stimuli elicited a response conflict, because they were also associated with the wrong response in the currently irrelevant task. It turned out that RR costs on task-switch trials were increased for incongruent compared to neutral stimuli. To explain this effect, they proposed the amplification of response conflict (ARC) account.

The ARC account interprets the reported interaction between congruency and response transition as an increase in response conflict if the response repeats compared to when it shifts. The account assumes that in task-switching contexts there is a general bias toward response shifting which facilitates RSs (cf. Hübner \& Druey, 2006). Consequently, on trials where the response has to be repeated, the wrong response has an activation advantage. Accordingly, on RR trials with incongruent stimuli the responseshift bias amplifies the response conflict already elicited by the stimulus. In contrast, on RS trials the response-shift bias promotes the activation of the correct response which should slightly reduce the response conflict. Together, the larger congruency effect on RR trials and the slightly smaller effect on RS trials result in more pronounced costs of RR for incongruent stimuli. Therefore, the assumption of a general response-shift bias in combination with the ARC account explains the increase in RR costs found for incongruent stimuli on task-switch trials (Grzyb \& Hübner, 2012a).

Yet, because the ARC account was tested with a rather specific sequential two-task procedure without task repetitions (for details see Grzyb \& Hübner, 2012a), its generality remained open. Moreover, Grzyb and Hübner (2012a) reasoned that the interaction between congruency and response transition might also be explained without assuming a general response-shift bias at least on task-switch trials. In the present study, therefore, the modulation of RR effects by congruency was examined again with an alternating-runs paradigm (Rogers \& Monsell, 1995) that also included task-repetition trials. As will be explained later, the assumption of a general response-shift bias predicts the same interaction between congruency and response transition when the task repeats, that is, a larger congruency effect on RR trials than on RS trials. In contrast, alternative explanations predict no or a reversed relationship on task-repetition trials. In the following, we outline the most prominent theories of $\mathrm{RR}$ effects in task switching, consider how they can explain Grzyb and Hübner's (2012a) results, and, 
finally, derive predictions for the present study, which are summarized in Table 1.

How can the different theories account for the basic RR cost on task-switch trials and what is their potential to explain its modulation by congruency? A first class of theories assumes that RR costs on task-switch trials reflect a strategic response-shift bias. This class includes the response-inhibition theory (e.g., Hübner \& Druey, 2006; Marí-Beffa, Cooper, \& Houghton, 2012), which assumes a general response-shift bias that is also present on taskrepetition trials, and the hierarchical-switching theory (Kleinsorge \& Heuer, 1999), which assumes a responseshift bias on task-switch trials only.

According to the response-inhibition theory, in contexts where flexible switching between tasks is necessary, the previous response is generally inhibited to prevent an erroneous re-execution of the same response on a different task (Druey \& Hübner, 2008; Grzyb \& Hübner, 2012b; Hübner \& Druey, 2006). Such a strategy would be especially beneficial when task switches are associated with response shifts as it is presumably the case in most everyday life situations (see also Cooper \& Marí-Beffa, 2008). The hierarchical switching theory assumes that tasks are hierarchically represented and that a switch at a high level of representation (e.g., the intended judgment) propagates downstream to subordinate levels including the response level (Kleinsorge \& Heuer, 1999). Accordingly, a switch at any higher level leads to a response-shift bias at the motor level (Kleinsorge, 1999). If the same response is required again, however, a re-switch is necessary at the response level, which produces RR costs. Obviously, theories assuming the existence of a response-shift bias on taskswitch trials are compatible with the ARC account. Therefore, they can easily explain the data of Grzyb and Hübner (2012a), as has been outlined before.

A second class of theories explains the RR effect by the modulation of the strength of associations and binding processes. Meiran (2000a, 2000b), for instance, assumed that after a category-response (C-R) association has been used to select a response, this association is strengthened, while the alternative association between the same response and the category of the irrelevant task is weakened. Consequently, if the task switches, a RR means that the weakened C-R association must be used for response selection, which produces costs compared to RSs where an unaltered C-R association selects the correct response. Similarly, Schuch and Koch (2004) suggested that during response selection the alternative C-R association is laterally inhibited and that this inhibition persists into the next trial. Closely related to these ideas is Altmann's (2011) suggestion that the repeating response serves as a retrieval cue for an episodic trace of the previous task performance. Costs arise in this case, because the retrieved episode only partially matches the present episode (see also, Hommel, Müsseler, Aschersleben, \& Prinz, 2001). However, Grzyb and Hübner (2012a) reasoned that episodic retrieval cannot explain the increase in RR costs for incongruent stimuli. In their study, on trials where the response repeated the only feature that repeated from the previous to the current episode was the response itself irrespective of the congruency of the

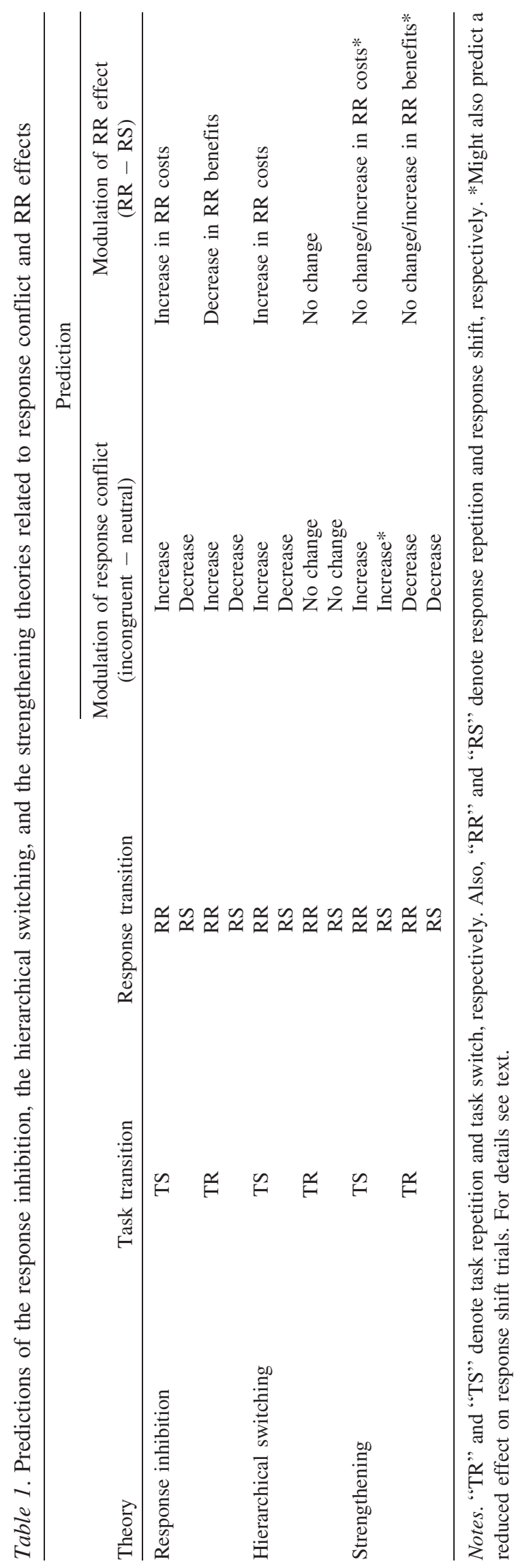


current stimulus. Thus, the mismatch should be the same for both stimulus types. On trials where the response shifted, in contrast, the mean mismatch should have been even larger for incongruent stimuli, because on one third of these trials a nontarget item could repeat from the previous to the current episode. Together, episodic retrieval would, if anything, predict the opposite pattern from what Grzyb and Hübner have found. Therefore, we will not consider this theory when deriving our hypothesis for the present study.

How would the strengthening and weakening of C-R associations alter the response conflict triggered by incongruent stimuli, and how would this modulate RR costs on task-switch trials? On such trials the response conflict should generally be increased. The repetition of a response means that the $\mathrm{C}-\mathrm{R}$ association that has been weakened on the previous trial is related to the now correct response. Thus, the correct response has to be selected by a weakened $\mathrm{C}-\mathrm{R}$ association while the wrong response can be activated by a C-R association that has not been weakened. Accordingly, the response conflict is increased for incongruent stimuli. In contrast, if the response shifts, then the C-R association related to the now wrong response has been strengthened on the previous trial. Therefore, the wrong response can be activated by a strengthened C-R association while the activation of the correct response is unchanged. Again, this results in an increase of the response conflict. Because an increased response conflict is predicted irrespective of response transition, the strengthening theories cannot explain a modulation of RR effects by congruency. However, with the additional assumptions that the altering of the C-R association that is related to the processing of the target and to the currently correct response has a larger impact on response conflict than the altering of the C-R association that is related to the currently wrong response, more specific predictions could be made. Then, the larger congruency effect on RR trials could be explained and, consequently, also the increase in RR costs for incongruent stimuli.

These considerations show that several theories are more or less compatible with the results of Grzyb and Hübner (2012a). Up to now, however, the ARC account has only been tested for task-switch trials in a specific paradigm. Therefore, the primary goal of the present study was to generalize the ARC account of Grzyb and Hübner (2012a) to a common task-switching paradigm and to task-repetition trials. In other words, we wanted to test if the assumption of a general response-shift bias is necessary to explain our results. To this end, we adapted the alternating-runs paradigm of Rogers and Monsell (1995), where participants have to switch between predictable runs of two alternative tasks. The classification of letters as consonants or vowels and the parity judgment of numerals served as tasks. Congruency was manipulated by always presenting a task-relevant stimulus item together with an irrelevant one. As in Grzyb and Hübner (2012a), we used incongruent and neutral stimuli. For incongruent stimuli the irrelevant item was always related to the irrelevant task and associated with the wrong response, whereas it was not associated with any task or response for neutral stimuli.

One might wonder why we did not include congruent stimuli, that is, stimuli whose irrelevant item is associated with the relevant task and the correct response. The inclusion of congruent stimuli would have allowed us to separately assess the effect of response conflict (as difference in performance between incongruent and congruent trials) and the effect of task priming (as difference in performance between congruent and neutral trials). With neutral and incongruent stimuli alone, the difference in performance reflects the sum of both effects (e.g., Steinhauser \& Hübner, 2007). However, one of our studies (Grzyb \& Hübner, 2012c), in which we also applied compound stimuli of numerals and letters, revealed that participants adapt their processing strategy to the set of involved stimuli. For instance, they responded faster to incongruent stimuli if, additionally to neutral stimuli, also congruent stimuli could occur. This was presumably an effect of perceptual discriminability. Incongruent and neutral stimuli can easily be discriminated. Therefore, if only these two stimulus types occur, then it is possible to respond more carefully, that is, slower, to conflict inducing stimuli than to neutral stimuli. However, if congruent stimuli, which perceptually are rather similar to incongruent stimuli, can also occur, then such a strategy is no longer applicable. Accordingly, Grzyb and Hübner (2012c) concluded that responses to incongruent stimuli are slowed down when congruent stimuli are absent, relative to when they are included.

Moreover, Grzyb and Hübner (2012c) found no or a very weak effect of response conflict (measured as incongruent vs. congruent) in the latencies, but again a reliable effect in the error rates. Thus, it seems that, when congruent stimuli are included in the design, the effect of response conflict induced by incongruent stimuli is moved more or less completely to the error rates, presumably by speeding up responses to these stimuli. This interpretation is also consistent with the fact that the Congruency $\times$ Response Transition interaction was no longer reliable in the latencies in conditions including congruent stimuli. Because the congruency effect in response times (RT) in Rogers \& Monsell's design (1995, Experiment 1) was also very small compared to task priming (and even nonexistent on taskrepetition trials), we assumed that similar strategic effects might play a role in their paradigm. Therefore, when adopting Rogers \& Monsell's procedure, we decided to use only neutral and incongruent stimuli so that we would be able to observe the Congruency $\times$ Response Transition interaction also in RT. ${ }^{1}$

A drawback of using incongruent and neutral stimuli is that we do not have an exact measure of response conflict. However, for the present objective the exact assessment of the size of response conflict was not a prerequisite, because we only needed to compare a response conflict condition with a no-conflict condition to test if the interaction between congruency and response transition is also present

1 Unfortunately, as the results show, this worked only for task switch trials. We will return to this issue in the Discussion. 
on task-repetition trials. In the remainder of the Introduction we will elaborate that such a result would support the existence of a general response-shift bias in task switching.

What are the predictions of the different theories for task-repetition trials (see Table 1)? The response-inhibition theory assumes that as on task-switch trials response inhibition induces a response-shift bias which can sometimes be observed as RR costs (e.g., Cooper \& Marí-Beffa, 2008; Marí-Beffa et al., 2012). Typically, however, on taskrepetition trials, this bias is not observed as costs, because other features of the previous trial repeat as well, most of which prime the correct response. According to the response-inhibition theory the positive effects of category priming (cf. Pashler \& Baylis, 1991) or of episodic retrieval (Altmann, 2011; Marí-Beffa et al., 2012) usually outweigh the negative effects of response inhibition, resulting in the observed RR benefits on task-repetition trials. Because a response-shift bias is assumed also on task-repetition trials, the ARC idea generalizes to these trials. Consequently, as for task-switch trials, an increased congruency effect on RR trials and a slightly decreased congruency effect on RS trials would be expected. Yet, because there are typically RR benefits on task-repetition trials, the modulation of the congruency effect should result in reduced RR benefits for incongruent compared to neutral stimuli on these trials. Put differently, irrespective of task transition RR effects should be shifted toward more costs (or less benefits) if the stimulus is incongruent.

Hierarchical switching, in contrast, does not assume a response-shift bias on task-repetition trials. According to this theory, RR benefits arise because RRs require no switch operation whereas RSs require one switch. Because no response-shift bias is assumed, the theory does neither predict a modulation of the congruency effect by response transition nor a modulation of RR benefits by congruency.

Finally, according to the strengthening theories, RR benefits on task-repetition trials arise, because a RR means that the strengthened C-R association can be used for response selection again producing benefits relative to RSs. Generally, these theories would predict a decreased congruency effect on task-repetition trials. That is, if the response repeats the $\mathrm{C}-\mathrm{R}$ association related to the now correct response has been strengthened on the previous trial. This decreases the response conflict elicited by incongruent stimuli. Conversely, if the response shifts, then the C-R association related to the now wrong response has been weakened on the previous trials. Again, this decreases the response conflict. However, as for task-shift trials, one can make the additional assumptions that the altering of the C-R associations that is related to the currently correct response has a larger impact on response conflict than the altering of the C-R association that is related to the currently wrong response. Then, one would expect a larger decrease of the congruency effect on RR trials and, consequently, also larger RR benefits for incongruent stimuli.

The different predictions are summarized in Table 1. Adopting the response-inhibition theory and the ARC account, we expected the congruency effects to be larger on RR trials than on RS trials. This relation should be independent of task transition. As a consequence, we expected RR costs on task-switch trials to be larger for incongruent stimuli than for neutral stimuli generalizing the findings of Grzyb and Hübner (2012a). Critically, on task-repetition trials, RR benefits should be smaller for incongruent stimuli than for neutral ones. Such a finding would support the existence of a general response-shift bias.

\section{Method}

\section{Participants}

Forty students (17 male; mean age 23.5 years) of the Universität Konstanz participated in the experiment. All participants reported normal or corrected-to-normal vision and were either paid 8 Euro per hour or fulfilled a course requirement.

\section{Apparatus}

The stimuli were presented on a 19-inch color monitor with a resolution of $1,280 \times 1,024$ pixels and a refresh rate of $60 \mathrm{~Hz}$. The viewing distance was approximately $50 \mathrm{~cm}$. A PC controlled stimulus presentation and response registration.

\section{Stimuli}

Stimuli were constructed using letters (G, K, M, R, A, E, I, $\mathrm{U})$ and numerals $(2,4,6,8,3,5,7,9)$ as stimulus items. There were also four neutral symbols $(*, \&, \%, ?)$ that were unrelated to both tasks. Stimuli consisted of a target and a distractor item, presented next to each other. The relative position of target and distractor was random. Stimuli were either neutral or incongruent, that is, the distractor was either drawn from the neutral set or from the character set of the currently irrelevant task (letters or numerals, respectively), such that target and distractor were associated with opposite responses. Stimuli were randomly drawn from a pool of all possible item combinations which was refilled when empty. We presented stimuli in white on a black background in an uppercase Courier New Bold font subtending a visual angle of approximately $1.1^{\circ}$ width and of $0.6^{\circ}$ height.

\section{Procedure}

During each experimental block a quadratic frame with four boxes was displayed. From trial to trial, stimuli were presented in a clockwise manner at the center of one of the boxes with the position indicating the relevant task. Participants performed the tasks in alternating runs (AABBAA ...). Accordingly, after a first cycle of four 
tasks the first box was reached again. This initial box was counterbalanced between participants. As tasks served the categorization of numbers as "even" or "odd" and the categorization of letters as "consonants" or "vowel." The result of a judgment had to be indicated by pressing one of two response buttons (left, right), which were the same for each task. The categories "even" and "consonant" were mapped to the left button, and the categories "odd" and "vowel" to the right button. Stimuli remained on screen until a response was given or until $5 \mathrm{~s}$ elapsed. The next trial started after $150 \mathrm{~ms}$. After an incorrect response, a short feedback tone $(500 \mathrm{~Hz}, 100 \mathrm{~ms})$ was presented and the response-stimulus interval was increased to $1,000 \mathrm{~ms}$. Participants were instructed to prepare for the upcoming tasks and to respond as fast as possible while trying to keep accuracy above $90 \%$.

The experiment encompassed 16 blocks of 64 trials each. At the end of each block speed and accuracy feedback was provided, and, if necessary, participants were reinstructed. Before the experimental blocks, participants practiced the tasks separately in five pure blocks for each task in which only neutral stimuli were presented. The pure blocks consisted of 32 trials each and alternated between letter and number task. Pure blocks and the first two experimental blocks were not analyzed. Also, the first four trials (one cycle) in each experimental block served as warm-up trials and were discarded from analysis.

\section{Design}

The experiment followed a within-participant design with task transition (task repetition, task switch), response transition (response repetition, response shift), and congruency (neutral, incongruent) as independent variables. Mean response times (RT) and error rates served as dependent variables. The effects of congruency and of RR were defined as incongruent minus neutral and response repetition minus response shift, respectively.

\section{Results}

Anticipatory responses (RT $<100 \mathrm{~ms}$ ) and extreme outliers larger than 4 standard deviations of the individual mean of each condition were removed from the data (less than $1.3 \%$ in each condition, cf. Ulrich \& Miller, 1994). We also wanted to eliminate sequential bottom-up effects, such as episodic retrieval (cf. Altmann, 2011; Marí-Beffa et al., 2012), which do not result from strengthening, response inhibition, or hierarchical switching but nevertheless might affect the interaction between response transition and congruency. To this end, we excluded trials where a stimulus item repeated from the previous $(n-1)$ to the current (n) trial $(\sim 20 \%)$. These exclusions included direct target repetitions, direct distractor repetitions, repetitions of the distractor on trial $n-1$ as target on trial n, and repetitions of the target on trial $n-1$ as distractor on trial $n$ (see also

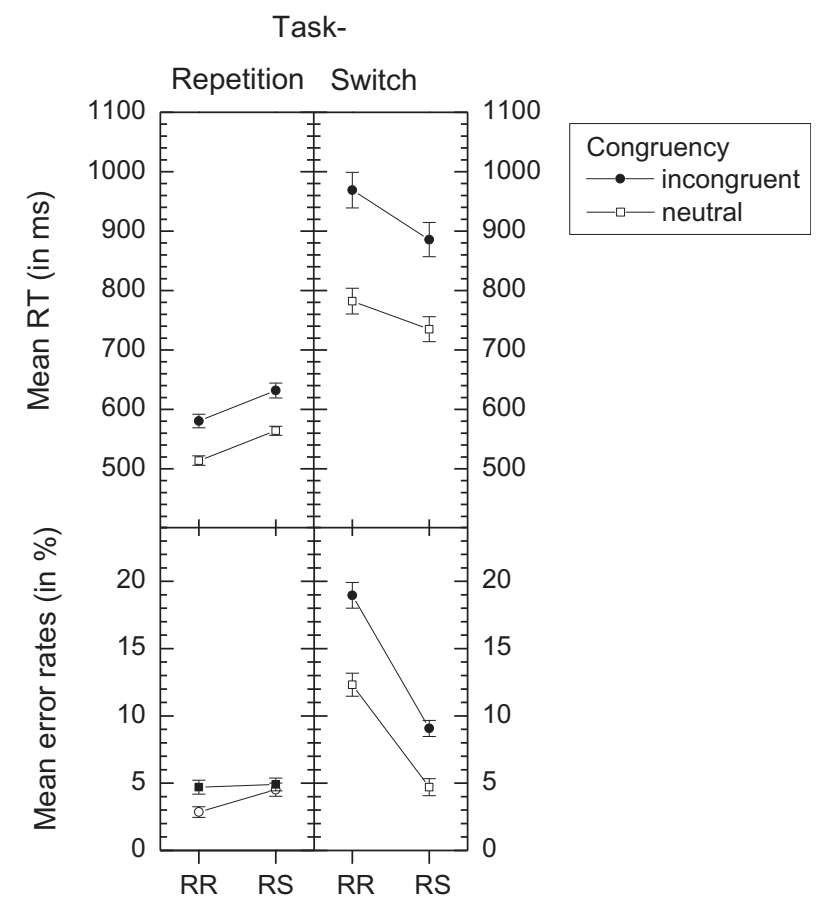

Figure 1. Mean response times (RT) and mean errors rates in neutral and incongruent trials as a function of task transition and response transition. "RR" and "RS" denote response repetition and response shift, respectively. Error bars indicate standard errors of the mean.

Juvina \& Taatgen, 2009; Mayr, Awh, \& Laurey, 2003). As a consequence, the amount of match or mismatch, for instance, depends only on the repetition or switch of the task and the response, thereby restricting any effect of episodic retrieval to the basic interaction between task transition and response transition. Finally, to make sure that we analyzed only those trials where participants followed the task sequence, trials preceded by an error in one $(n-1)$ or two $(n-2)$ trials before were excluded from the analysis $(14.8 \%)$. These measures did not affect the overall results most critically, they did not qualitatively change the interaction between response transition and congruency. Results are depicted in Figure 1 and listed in Table 2.

\section{Response Times}

Mean latencies of correct responses were entered into a three-way ANOVA with the independent variables task transition (task repetition, task switch), response transition (response repetition, response shift), and congruency (neutral, incongruent), all realized within participants. The analysis revealed significant main effects of task transition, $F(1,39)=201, \quad p<.001$, and congruency, $F(1,39)=$ $265, p<.001$, which, however, were qualified by several interactions. First, we found the typical interaction between task transition and response transition, $F(1,39)=163$, $p<.001$. On task-repetition trials RR produced benefits 
Table 2. Mean response times and mean error rates for the different conditions

\begin{tabular}{lllrr}
\hline Task transition & Response transition & Congruency & RT $(\mathrm{ms})$ & Mean error rates $(\%)$ \\
\hline Task shift & Response repetition & Incongruent & $969(30)$ & $19.0(0.96)$ \\
& & Neutral & $782(22)$ & $12.3(0.86)$ \\
& Response shift & Incongruent & $886(29)$ & $9.07(0.60)$ \\
& & Neutral & $735(21)$ & $4.70(0.63)$ \\
Task repetition & Response repetition & Incongruent & $580(11)$ & $4.70(0.52)$ \\
& & Neutral & $514(8.0)$ & $2.86(0.40)$ \\
& Response shift & Incongruent & $632(13)$ & $4.90(0.48)$ \\
& & Neutral & $564(7.7)$ & $4.51(0.49)$ \\
\hline
\end{tabular}

Notes. RT = mean response time. The values in the parentheses give the corresponding standard error of the mean.

( $-51 \mathrm{~ms})$, whereas they produced costs $(65 \mathrm{~ms})$ on taskswitch trials. Second, there was a two-way interaction between task transition and congruency, $F(1,39)=152$, $p<.001$. Task-shift costs were larger for incongruent (322 ms) than for neutral stimuli (220 ms). Third, the interaction between response transition and congruency was also significant, $F(1,39)=9.51, p<.01$. However, these interactions were further qualified by a three-way interaction between all independent variables, $F(1,39)=7.61$, $p<.01$. Further analyses revealed that the Response Transition $\times$ Congruency interaction was reliable on task-shift trials, $F(1,39)=10.1, p<.01$, but not on task-repetition trials, $F(1,39)<1, p>.80$. This means that, if the task shifted, the congruency effect was larger on RR trials (187 ms) than on RS trials (151 ms). In contrast, on taskrepetition trials the congruency effect was independent of response transition (RR $67 \mathrm{~ms}$, RS $68 \mathrm{~ms}$ ). To put it the other way, on task-shift trials, RR costs were larger for incongruent stimuli $(83 \mathrm{~ms})$ than for neutral ones (47 ms), whereas there was no such difference in RR benefits on task-repetition trials (neutral $-50 \mathrm{~ms}$, incongruent $-52 \mathrm{~ms}$ ).

\section{Error Rates}

Mean error rates for responses were subjected to an ANOVA of the same type as for the latencies. The significant main effects of task transition, $F(1,39)=161$, $p<.001$, response transition, $F(1,39)=89.0, p<.001$, and congruency, $F(1,39)=36.4, p<.001$, were qualified by several two-way interaction. First, the Task Transition $\times$ Response Transition interaction was significant, $F(1,39)=115, p<.001$, indicating that there were rather small RR benefits $(-0.93 \%)$ on task-repetition trials, but substantial RR costs $(8.75 \%)$ on task-shift trials. Second, the reliable interaction between task transition and congruency, $F(1,39)=47.6, p<.001$, reflects that task-shift costs were larger for incongruent stimuli $(9.21 \%)$ than for neutral stimuli $(4.82 \%)$. Third, the critical interaction between response transition and congruency was also significant, $F(1,39)=11.1, p<.01$. It indicates that the congruency effect was larger on RR trials (4.24\%) than on RS trials $(2.38 \%)$. In terms of RR effects, this interaction means that RR costs were larger for incongruent stimuli
$(4.85 \%)$ than for neutral ones $(2.98 \%)$. The three-way interaction between all three variables was far from significant, $F(1,39)<1, \quad p>.49$. The non-significant three-way interaction does not necessarily imply that the Response Transition $\times$ Congruency interaction was significant on task-repetition trials alone. However, the interaction effect on task-repetition trials was critical to our objective. Therefore, we tested the corresponding contrast which was significant, $F(1,39)=11.1, p<.01$. Also on taskrepetition trials alone the congruency effect was the larger when the response repeated $(1.84 \%)$ than when the response shifted $(0.39 \%)$.

\section{Discussion}

In the present experiment we tested the generality of the ARC account, recently proposed by Grzyb and Hübner (2012a), explaining the modulation of RR effects in task switching by the congruency of the actual stimulus. These researchers observed that RR costs were larger for incongruent stimuli than for neutral ones. They concluded that a general response-shift bias present in task switching amplifies the response conflict on trials where the response repeats and slightly reduces response conflict on trials where the response shifts. However, in that study a specific sequential two-task procedure was used, and there were no task repetitions. Thus, in the present study we applied a common alternating-runs paradigm (Rogers \& Monsell, 1995), where the tasks switched on every other trial.

Our results revealed that the interaction between congruency and the transition of responses is not restricted to task-switch trials. When the response repeated, congruency effects were larger irrespective of task transition, at least in the error rates. This also means that RR effects were modulated by congruency. Whereas on task-shift trials RR costs were larger for incongruent stimuli than for neutral stimuli, on task-repetition trials, RR benefits were reduced for incongruent stimuli, although this latter effect was present only in the error rates. Moreover, our results indicate that response conflict is reduced if the response shifts. This is supported by our finding that on task-repetition trials the congruency effect was practically absent in error rates $(0.39 \%)$ when the response shifted. 
Our results can easily be interpreted by the ARC account if one assumes that there is a general response-shift bias on task-switch and on task-repetition trials alike due to the inhibition of the previous response. In contrast, our results cannot be fully explained by hierarchical switching or the strengthening theories. Whereas hierarchical switching predicts no modulation at all of RR benefits on task-repetition trials by congruency, ${ }^{2}$ the strengthening theories would assume that RR benefits should be larger for incongruent stimuli. However, we found smaller RR benefits on task-repetition trials for incongruent stimuli compared to neutral stimuli in the error rates. Nevertheless, RR effects in task switching seem to be a complex phenomenon that presumably involves several processes (Grzyb \& Hübner, 2012a). Thus, although the present results clearly support the existence of a general response-shift bias in task switching, they do not falsify the existence of other mechanisms contributing to RR effects.

Why was the decrease of RR benefits present only in the error rates? One possible reason is that excluding congruent stimuli from the design did not prevent speed-accuracy tradeoffs and strategic processing of incongruent stimuli in the way we hoped. As other experiments in our laboratory indicate, the occurrence of congruent stimuli moves the effects of response conflict from RT to the error rates. Consequently, evidence for $\mathrm{ARC}$ is also only present in the error rates (Grzyb \& Hübner, 2012c). This was the reason for not including congruent stimuli in the present experiment. Although our selection of stimuli had the intended effect that the investigated interaction between congruency and response transition was present in both error rates and $\mathrm{RT}$ on task-switch trials, it failed with respect to task-repetition trials. Accordingly, we think that despite the exclusion of congruent stimuli response conflict had no or a minor effect on RT on task-repetition trials. Consequently, this effect could not interact with the response-shift bias which explains the missing interaction in RT.

Speed-accuracy tradeoffs and similar strategies might also be the reason why other authors did not find a reliable interaction between congruency and the transition of responses (Schuch \& Koch, 2004). Schuch and Koch (2004), for instance, used a different experimental paradigm where, on every trial, participants had to switch the task from a first to a second of two consecutively presented stimuli. When examining the Congruency $\times$ Response Transition interaction, the authors found inconsistent results (Schuch \& Koch, 2004, p. 577). For three experiments with congruent and incongruent stimuli they report RR costs separately for these stimulus types. In their Experiment 1 they found numerically larger RR costs for incongruent stimuli in the error rates but no difference in RT, whereas in Experiment 3B RR costs were numerically larger for incongruent stimuli in error rates but significantly reduced in RT. Finally, in Experiment 3B, RR costs were significantly larger for incongruent stimuli in the error rates and numerical larger in RT. Thus, in all three experiments
$\mathrm{RR}$ costs in the error rates were larger for incongruent than for congruent stimuli, at least numerically. In RT, however, the results were mixed. Nevertheless, Schuch and Koch's (2004) results are in line with other results indicating that the Congruency $\times$ Response Transition interaction is more stable in the error rates than in RT, presumably because additional strategic effects mask its existence when congruent stimuli are introduced in the paradigm (Grzyb \& Hübner, 2012c). Another important difference between Schuch and Koch's (2004) and the present study is the sample size. Whereas Schuch and Koch had only 12 participants in each experiment, we analyzed the data of 40 participants. Thus, not only was the statistical power of the present study larger, but also the likelihood smaller that individual processing strategies would dilute our results.

Taken together, our results generalize Grzyb and Hübner's (2012a) findings and their ARC account to a common task-switching paradigm (cf. Kiesel et al., 2010) including task-repetition trials. Thus, at least some of the variation of RR effects reported in the literature seems to have been due to modulations by congruency. Moreover, the modulation of RR effects by congruency is not only of interest in its own, but also for model selection. Our results clearly support the existence of a general response-shift bias in task switching and are hard to explain by other theories. Yet, the whole pattern of RR benefits on task-repetition trials and that of RR costs on task-shift trials are only explained by the interaction of several processes. First, in task-switching contexts a general response-shift bias is strategically implemented (Cooper \& Marí-Beffa, 2008; Hübner \& Druey, 2006; Marí-Beffa et al., 2012). This bias is presumably realized by the inhibition of the previous response (Hübner \& Druey, 2006), which produces RR costs on task-shift trials. The inhibition amplifies response conflicts on RR trials, which is reflected by larger congruency effect on RR trials. Second, the repetition of stimulus and task features (e.g., stimulus category or feature overlap between processing episodes) produces positive effects on performance. Accordingly, RR benefits are usually observed on task-repetition trials, because the positive effects of, for instance, category priming (cf. Pashler \& Baylis, 1991), or episodic retrieval (Altmann, 2011; Marí-Beffa et al., 2012), outweigh the negative effect of response inhibition.

\section{Acknowledgments}

This research was supported by a grant $(\mathrm{Hu} 432 / 9)$ from the German Research Foundation (Deutsche Forschungsgemeinschaft DFG) to Ronald Hübner.

\section{References}

Altmann, E. M. (2011). Testing probability matching and episodic retrieval accounts of response repetition effects in task switching. Journal of Experimental Psychology:

2 Although this prediction is consistent with the RT data, it is incompatible with the error rates data. 
Learning, Memory and Cognition, 37, 935 951. doi: $10.1037 / \mathrm{a} 0022931$

Cooper, S., \& Marí Beffa, P. (2008). The role of response repetition in task switching. Journal of Experimental Psychology: Human Perception and Performance, 34, 11981211.

Druey, M. D., \& Hübner, R. (2008). Response inhibition under task switching: Its strength depends on the amount of task irrelevant response activation. Psychological Research, 72, 515527.

Grzyb, K. R., \& Hübner, R. (2012a). Excessive response repetition costs under task switching: How response inhibi tion amplifies response conflict. Journal of Experimental Psychology: Learning, Memory and Cognition. Advance online publication. doi: 10.1037/a0028477

Grzyb, K. R., \& Hübner, R. (2012b). Response repetition costs in task switching: How they are modulated by previous trial response category activation. Acta Psychologica, 139, 97 103. doi: 10.1016/j.actpsy.2011.10.006

Grzyb, K. R., \& Hübner, R. (2012c). Strategic modulation of response inhibition in task switching. Manuscript in preparation.

Hommel, B., Müsseler, J., Aschersleben, G., \& Prinz, W. (2001). The Theory of Event Coding (TEC): A framework for perception and action planning. Behavioral and Brain Sciences, 24, 849878.

Hübner, R., \& Druey, M. D. (2006). Response execution, selection, or activation: What is sufficient for response related repetition effects under task shifting? Psychological Research, 70, 245261.

Juvina, I., \& Taatgen, N. A. (2009). A repetition suppression account of between trial effects in a modified Stroop paradigm. Acta Psychologica, 131, 72 84. doi: 10.1016/ j.actpsy.2009.03.002

Kiesel, A., Steinhauser, M., Wendt, M., Falkenstein, M., Jost, K., Philipp, A. M., \& Koch, I. (2010). Control and interference in task switching a review. Psychological Bulletin, 136, 849874.

Kleinsorge, T. (1999). Response repetition benefits and costs. Acta Psychologica, 103, 295310.

Kleinsorge, T., \& Heuer, H. (1999). Hierarchical switching in a multi dimensional task space. Psychological Research, 62, 300312.

Koch, I., Schuch, S., Vu, K. P., \& Proctor, R. W. (2011). Response repetition effects in task switching: Dissociating effects of anatomical and spatial response discriminability. Acta Psycho logica, 136, 399 404. doi: 10.1016/j.actpsy.2011.01.006
Marí Beffa, P., Cooper, S., \& Houghton, G. (2012). Unmixing the mixing cost: Contributions from dimensional relevance and stimulus response suppression. Journal of Experimental Psychology: Human, Perception and Performance, 38, 478 488. doi: 10.1037/a0025979

Mayr, U., Awh, E., \& Laurey, P. (2003). Conflict adaption effects in the absence of executive control. Nature Neuro science, 6, 450452.

Meiran, N. (2000a). Modeling cognitive control in task switch ing. Psychological Research, 63, 234249.

Meiran, N. (2000b). Reconfiguration of stimulus task sets and response task sets during task switching. In S. Monsell \& J. Driver (Eds.), Control of cognitive processes: Attention and performance XVIII (pp. 377 399). Cambridge, MA: MIT Press.

Pashler, H., \& Baylis, G. (1991). Procedural learning: 2. Intertrial repetition effects in speeded choice tasks. Journal of Experimental Psychology: Learning, Memory and Cog nition, 17, 3348.

Rogers, R. D., \& Monsell, S. (1995). Costs of a predictable switch between simple cognitive tasks. Journal of Experi mental Psychology: General, 124, 207231.

Schuch, S., \& Koch, I. (2004). The costs of changing the representation of action: Response repetition and response response compatibility in dual tasks. Journal of Experimental Psychology: Human Perception and Performance, 30, 566582.

Steinhauser, M., \& Hübner, R. (2007). Automatic activation of task related representations in task shifting. Memory \& Cognition, 35, 138155.

Ulrich, R., \& Miller, J. (1994). Effects of truncation on reaction time analysis. Journal of Experimental Psychology: General, 123, 3480.

Kai Robin Grzyb

Fachbereich Psychologie

Universität Konstanz

Fach D29

78457 Konstanz

Germany

Tel. +497531883153

E mail kairobin.grzyb@uni konstanz.de 